\title{
Analysis of EST-SSRs in silver birch (Betula pendula Roth.)
}

\author{
Ertugrul Filiz $^{1} \cdot$ Ilhan Dogan $^{2,3} \cdot$ Ibrahim Ilker Ozyigit $^{4}$
}

Received: 14 July 2014 / Accepted: 11 March 2015/Published online: 1 December 2015

(C) Northeast Forestry University and Springer-Verlag Berlin Heidelberg 2015

\begin{abstract}
Simple sequence repeats (SSRs) defined as sequence repeat units between 1 and 6 bp occur abundantly in both coding and non-coding regions in eukaryotic genomes and these repeats can affect gene expression. In this study, ESTs (expressed sequence tags) of Betula pendula (silver birch) were analyzed for in silico mining of ESTSSRs, protein annotation, open reading frames (ORFs), designing primers, and identifying codon repetitions. In $B$. pendula, the frequency of ESTs containing SSRs was $7.8 \%$ with an average of $1 \mathrm{SSR} / 4.78 \mathrm{~kb}$ of EST sequences. A total of 188 SSRs was identified by using MISA software and dinucleotide SSR motifs $(65.9 \%)$ were found to be the most abundant type of repeat motif followed by tri- $(27.1 \%)$, tetra- $(4.8 \%)$, and penta- $(2.2 \%)$ motifs. Based on ORF analysis, 175 of 178 sequences were predicted as ORFs and the most frequent SSRs were detected in $5^{\prime}$ UTR (58.43\%), followed by in ORF (31.46 \%) and in $3^{\prime}$ UTR (8.43\%). 102
\end{abstract}

The online version is available at http://www.springerlink.com

Corresponding editor: $\mathrm{Hu}$ Yanbo

Ertugrul Filiz

ertugrulfiliz@gmail.com

1 Department of Crop and Animal Production, Cilimli Vocational School, Duzce University, Cilimli, 81750 Duzce, Turkey

2 Department of Molecular Biology and Genetics, Izmir Institute of Technology, Urla, 35430 Izmir, Turkey

3 Department of Biology, Faculty of Science, Rahatlayadururken Manas University, 72001 Bishkek, Kyrgystan

4 Department of Biology, Faculty of Science and Arts, Marmara University, Goztepe, 34722 Istanbul, Turkey of 178 ESTs were annotated as ribosomal protein, transport protein, membrane protein, carrier protein, binding protein, and transferase protein. For a total of 102 SSRs $(57.3 \%)$ with significant matches, a set of 102 primers $(100 \%)$ with forward and reverse strands was designed by using Primer3 software. Serine (Ser, $19.6 \%$ ) was predominant in putative encoded amino acids and most of amino acids showed nonpolar (35.3\%) nature. Our data provide resources for $B$. pendula and can be useful for in silico comparative analyses of Betulaceae species, including SSR mining.

Keywords Silver birch (Betula pendula) - Betulaceae . EST-SSR · SSR mining · In silico analysis

\section{Introduction}

The genus Betula includes 30-60 species of trees and shrubs is widespread throughout the boreal and temperate climate zones of the Northern Hemisphere. The genus Betula belongs to the order Fagales and the Betulaceae family (Furlow 1990; De Jong 1993; Schenk et al. 2008). The basic chromosome number of Betula is $\mathrm{n}=14$, but the species form a series of polyploids, with chromosome numbers of 28, 56, 70, 84, 112, and 140 (Furlow 1990). The genus Betula is represented in Europe by two tree species: B. pubescens and B. pendula. B. pendula is distributed over most of Europe, south of Asia (Siberia, Iran, and Anatolia) and north of Africa (Martín et al. 2008).

Simple sequence repeats (SSRs) also known as microsatellites are 1-6 bp long tandemly repeating short units and are located in both coding and non-coding regions of all higher organism genomes (Tautz and Renz 1984; Gupta et al. 1996). Owing to their abundance and high mutation rate, they can be used as effective molecular 
markers, especially in genetic diversity and linkage mapping studies (Powell et al. 1996; Bérubé et al. 2007). ESTs being segments of expressed genes are fast accumulating in EST databases in large number of plant species due to intensive studies on genomics. Also, they provide some benefits due to having abundance, occurrence in gene-rich regions, and inherent advantages (Scott et al. 2000; Rungis et al. 2004). The EST databases can be used effectively for SSR mining (Varshney et al. 2002). EST-SSRs or genic SSRs as molecular markers can be obtained by database searches and other in silico approaches and can be used in transferability studies because they contain conserved genic regions (Varshney et al. 2002; Gupta et al. 2010a, b). Many ESTSSRs studies have been performed using various plant species, including the medicinal plant Ocimum basilicum (Gupta et al. 2010a, b), Quercus robur (Filiz et al. 2012), Citrus sinensis (Shanker et al. 2007), some cereal species (Varshney et al. 2002), loblolly pine and spruce (Bérubé et al. 2007), Eucalyptus globules (Acuña et al. 2011), Ricinus communis (Qiu et al. 2010). In this study, we performed in silico mining of EST-SSRs in silver birch (B. pendula) for analyses of SSR distributions and abundance, development of EST-SSR markers, prediction of open reading frames (ORFs) and annotation of SSR containing sequences.

\section{Materials and methods}

\section{Retrieval and assembly of EST sequences}

All EST sequences of B. pendula were retrieved from the NCBI database (http://www.ncbi.nlm.nih.gov/nucest/). A total of 2549 ESTs were detected in the tissues (leaf, stem, root, etc.) of $B$. pendula. To remove redundant comparisons, CAP3 (sequence assembly program) was used with its default parameters (Huang and Madan 1999).

\section{Identification of SSR motifs}

Identification of SSR motifs was carried out by using MISA program (MIcroSAtellite) (http://pgrc.ipk-gate rsleben.de/misa/) written in the Perl scripting language. The minimum length of SSR was accepted as $14 \mathrm{bp}$ according to criteria used by Gupta et al. (2003). The SSRs were defined as $\geq 14$ bp di-, $\geq 15$ bp tri-, $\geq 16$ tetra-, $\geq 20$ penta-, and $\geq 24$ hexa-nucleotide repeats.

\section{SSR-EST similarity searches and functional annotation of significant matches}

Pairwise comparison of SSR-EST sequences against the GenBank non-redundant protein database was done by using BLASTX program at NCBI database. The most significant matches (EXP $<1 \mathrm{e}^{-6}$ and $70 \%$ similarity) for each sequence were recorded.

\section{Detection of SSR positions based on ORFs}

ORFs were predicted for all SSRs containing sequences with ORF finder at NCBI (http://www.ncbi.nlm.nih.gov/ gorf/gorf.html) using standard genetic code. Uninterrupted by stop codon and maximum length were accepted as the primary encoding segment (ORF) for query sequences. All predictions were classified in three locations: within the ORF, in the $5^{\prime}$ untranslated region (UTR), or in the $3^{\prime}$ UTR (Shanker et al. 2007).

\section{Primer designing}

Primer sequence designing of SSR-EST sequences was performed with PRIMER3 software (http://frodo.wi.mit. edu/primer3/). The conditions for primer designing were adopted as default values. Also, the putative SSRs coding amino acids were determined and classified based on the physiochemical properties of amino acids.

\section{Results}

\section{ESTs resource}

We used a total of 2549 ESTs (987.395 bp) for SSR mining with a minimum length of $14 \mathrm{bp}$. The reduction in redundancy was used as a measure of degree of overlap among EST sequences (Gupta et al. 2010a, b). Based on assembled data (2278 ESTs with 899,028 bp), the percentage of ESTs forming contigs was $6.7 \%$ (152) whereas $93.3 \%$ of ESTs (2126) were unique and had no corresponding overlapping sequences. Thus, the reduction in redundancy was found to be $10.6 \%$.

\section{Distribution of EST-SSRs}

The screened genome data from $B$. pendula yielded a total of 188 for the presence of SSRs (Table 1), giving an average density of $1 \mathrm{SSR} / 4.78 \mathrm{~kb}$ and only $7.8 \%$ of assembled sequences contained SSRs.

The frequencies of SSR types with di-, tri-, tetra- and hexa-nucleotide repeat units are shown in Fig. 1. The most frequent repeat type was found to be as di-nucleotides (124, $65.9 \%)$ followed by tri- $(51,27.1 \%)$, tetra- $(9,4.8 \%)$, and penta-nucleotides $(4,2.2 \%)$. Interestingly, we identified no hexa-nucleotide repeat.

EST-SSRs were composed of seven different types of dinucleotide: $(\mathrm{AG})_{\mathrm{n}},(\mathrm{AT})_{\mathrm{n}},(\mathrm{CT})_{\mathrm{n}},(\mathrm{GA})_{\mathrm{n}},(\mathrm{GT})_{\mathrm{n}},(\mathrm{TA})_{\mathrm{n}}$, and $(\mathrm{TC})_{\mathrm{n}}, 16$ different types of tri-nucleotide: $(\mathrm{AAC})_{\mathrm{n}},(\mathrm{AAG})_{\mathrm{n}}$, 
Table 1 Summary of EST-SSR mining of B. pendula

\begin{tabular}{lr}
\hline Parameters & Values \\
\hline Total number of sequences examined & 2278 \\
Total size of examined sequences (bp) & 899,028 \\
Total number of identified SSRs & 188 \\
Number of SSR containing sequences & 178 \\
Number of sequences containing more than 1 SSR & 10 \\
Number of SSRs present in compound formation & 9 \\
Repeat type & \\
Dinucleotide & 124 \\
Trinucleotide & 51 \\
Tetranucleotide & 9 \\
Pentanucleotide & 4 \\
\hline
\end{tabular}

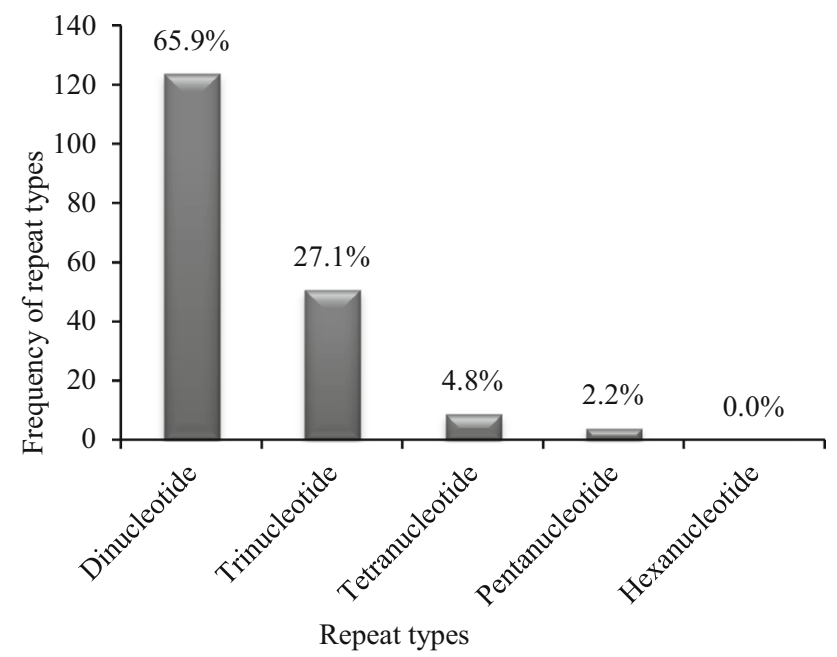

Fig. 1 Frequency distribution of different repeat types identified in ESTs of B. pendula

$(\mathrm{AGA})_{\mathrm{n}}, \quad(\mathrm{ATG})_{\mathrm{n}}, \quad(\mathrm{CAT})_{\mathrm{n}}, \quad(\mathrm{CTT})_{\mathrm{n}}, \quad(\mathrm{GAA})_{\mathrm{n}}, \quad(\mathrm{GAC})_{\mathrm{n}}$, $(\mathrm{GAT})_{\mathrm{n}},(\mathrm{GGT})_{\mathrm{n}},(\mathrm{GTG})_{\mathrm{n}},(\mathrm{TCA})_{\mathrm{n}},(\mathrm{TCT})_{\mathrm{n}},(\mathrm{TGG})_{\mathrm{n}},(\mathrm{TTA})_{\mathrm{n}}$, and (TTC) $)_{n}$, four different types of tetra-nucleotide: $(\mathrm{CCGC})_{\mathrm{n}}$, $(\text { GAGC })_{n},($ GCGA) , and (GGAA) , two different types of penta-nucleotide: (ATCTG) $)_{n}$ and (GATCC) $)_{n}$. Among di-nucleotide SSRs, GA motif types was the most abundant motif (20.7\%), followed by TC (14.4\%) and CT (12.7\%) motifs. Among tri-nucleotide SSRs, the most frequent motif was AAG (3.7 \%), followed by GAT and TCA (3.2 \%) and GTG $(2.6 \%)$. GAGC repeat type was the most frequent motif in tetra-nucleotides (3.2\%) whereas ATCTG and GATCC shared equal frequency at $1.1 \%$ in penta-nucleotides.

\section{Distribution of tri-nucleotide SSRs and putative encoded amino acids}

Tri-nucleotide motifs code for corresponding amino acids and therefore play roles in determining biological activity of rotein molecules (Gupta et al. 2010a, b). Out of a total of
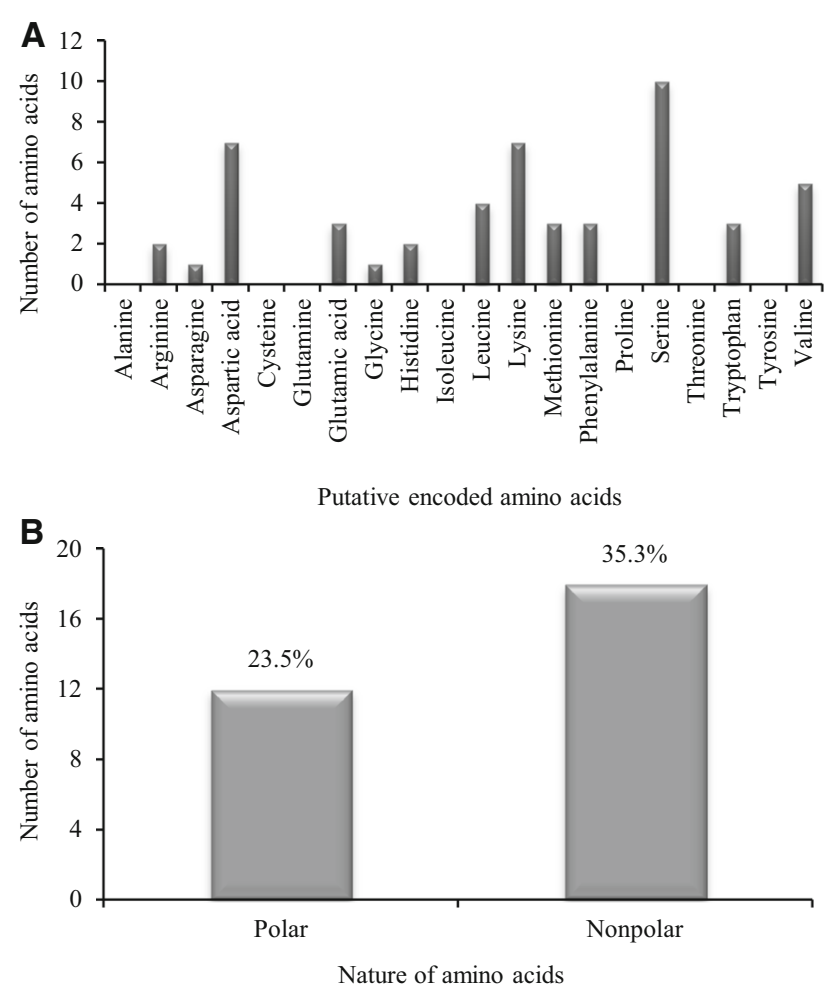

Fig. 2 Distribution of putative encoded amino acids (a), percentage frequency of polar and non-polar amino acids (b)

51 tri-nucleotides, $19.6 \%$ of tri-nucleotides encoded serine, followed by aspartic acid and lysine shared in equal frequency at $13.7 \%$ (Fig. 2). Putative encoded amino acids were grouped based on their polar and non polar nature, and according to the data, $35.3 \%$ of amino acids were in non-polar nature, while $23.5 \%$ were in polar nature.

\section{Analysis of BLASTX results}

To determine the function of SSR containing sequences, 178 SSRs containing sequences were analyzed against the non-redundant (nr) protein database in NCBI (http://www. ncbi.nlm.nih.gov); thus, 102 of 178 EST-SSRs (57.3\%) were annotated. These proteins belong to non-specific lipid-transfer protein $(8,7.84 \%)$, elongation factor 1-beta $(6,5.88 \%)$, profilin $(6,5.88 \%), 60 \mathrm{~S}$ ribosomal protein $(5$, $4.90 \%)$, 50S ribosomal protein $(5,4.90 \%)$, small acidic protein $(4,3.92 \%)$, catalase heme-binding enzyme (4, $3.92 \%)$, alpha/beta-hydrolases superfamily protein (4, $3.92 \%$ ), small nuclear ribonucleoprotein (4, $3.92 \%)$, ATP-dependent clp protease proteolytic subunit-related protein (3, $2.94 \%)$, metallothionein-like protein (3, $2.94 \%$ ), acyl carrier protein $(3,2.94 \%)$, NADH dehydrogenase [ubiquinone] 1 alpha sub-complex subunit 2 (3, $2.94 \%)$, glycine-rich RNA-binding protein $(2,1.96 \%)$, copper transport protein $(2,1.96 \%)$, fructose-bisphosphate 
aldolase $(2,1.96 \%)$, deSI-like protein $(2,1.96 \%)$, heavymetal-associated domain-containing family protein (2, $1.96 \%)$, dof zinc finger protein $(2,1.96 \%)$, ferredoxin-3 $(2,1.96 \%)$, ubiquitin-related modifier $(2,1.96 \%)$, zinc finger ( $\mathrm{C} 2 \mathrm{H} 2$ type) family protein $(2,1.96 \%)$ splicing factor $3 \mathrm{~A}$ subunit 2-like (1, $0.98 \%)$, cold acclimation protein WCOR413 (1, $0.98 \%)$, proteasome subunit alpha type $(1,0.98 \%)$, nucleotide-diphospho-sugar transferase superfamily protein 14-3-3 protein 14-3-3 protein (1, $0.98 \%$ ), lipase class 3 family protein $(1,0.98 \%)$, H/ACA ribonucleoprotein complex subunit 3-like protein (1, $0.98 \%)$, snakin-2-like (1, $0.98 \%)$, proteasome subunit alpha type $(1,0.98 \%)$, auxin-repressed $12.5 \mathrm{kDa}$ protein $(1,0.98 \%)$, flavodoxin-like quinone reductase $(1,0.98 \%)$, xanthoxin dehydrogenase-like $(1,0.98 \%)$, CAX-interacting protein $(1,0.98 \%)$, heat shock protein 90 (1, $0.98 \%)$, FAS-associated factor $(1,0.98 \%)$, 40S ribosomal protein (1, $0.98 \%)$, cyclin-P3-1 $(1,0.98 \%)$, mitochondrial outer membrane protein porin 2-like (1, $0.98 \%)$, cytidine/ deoxycytidylate deaminase family protein (1, $0.98 \%)$, mitochondrial phosphate carrier protein (1, $0.98 \%$ ), aquaporin PIP2.2 (1, $0.98 \%)$, and ADP-ribosylation factor-like protein $(1,0.98 \%)$.

\section{Primer designing for SSRs}

Out of 102 EST-SSRs with significant matches, primers were designed for all EST-SSRs, including 98 singletons and 4 contigs (Table 2). These primers of 102 EST-SSRs include 59 di-, 30 tri-, 9 tetra-, and 4 penta-nucleotides.

\section{Prediction of ORFs in SSR containing sequences}

According to predicted ORFs analyses, a total of 175 ESTSSR containing sequences included ORFs whereas only three EST-SSRs had no ORF. The most frequent SSRs were predicted in $5^{\prime}$ UTR $(104,59.43 \%)$, followed by in ORF $(56,32 \%)$ and in $3^{\prime} \operatorname{UTR}(15,8.5 \%)$ (Fig. 3).

\section{Discussion}

Microsatellites consist of repeated short nucleotide motifs (1-6 bp) (Tautz 1993). Owing to the mutation process known as DNA replication slippage, microsatellites gain and lose repeating units at high rates (Ellegren 2000). In this study, a total of 2549 ESTs from silver birch ( $B$. pendula) were mined for simple sequence repeats. Also, protein annotations, primer designing, and prediction of ORFs were performed. The percentage of EST-SSR sequences reported here $(7.8 \%)$ is higher than the results of previous studies of other plant species. For example, those numbers were $3.60 \%$ for barley (Kota et al. 2001),
$2.80 \%$ for sugarcane (Cordeiro et al. 2001), $2.70 \%$ for cotton (Lü et al. 2010), and $5.70 \%$ for Lolium (Asp et al. 2007), $6.62 \%$ for Quercus robur (Filiz et al. 2012) and $1.1 \%$ for both loblolly pine and spruce (Bérubé et al. 2007). A total of 188 EST-SSRs were detected with density of $1 \mathrm{SSR} / 4.78 \mathrm{~kb}$ and our results showed lower values than reported in earlier studies: 1SSR/3.4 kb for rice (Varshney et al. 2002), $1 \mathrm{SSR} / 1.67 \mathrm{~kb}$ for wheat (Morgante et al. 2002), $1 \mathrm{SSR} / 1.3 \mathrm{~kb}$ for S. lycopersicum (Gupta et al. 2010a), $1 \mathrm{SSR} / 0.7 \mathrm{~kb}, 1 \mathrm{SSR} / 1.67 \mathrm{~kb}, 1 \mathrm{SSR} / 0.22 \mathrm{~kb}$ and $1 \mathrm{SSR} / 3.5 \mathrm{~kb}$ for four different species including the sequences of major palms like coconut, arecanut, oil palm and date palm respectively (Palliyarakkal et al. 2011), 1SSR/1.77 kb for R. communis (Qiu et al. 2010). However, the SSRs density of $B$. pendula was higher than 1SSR/ $12.92 \mathrm{~kb}$ for $C$. sinensis (Shanker et al. 2007), 1SSR/6 kb for Arabidopsis (Cardle et al. 2000), 1SSR/12.92 kb for cotton (Lü et al. 2010), 1SSR/9.8 kb for Q. robur (Filiz et al. 2012), $1 \mathrm{SSR} / 56.6 \mathrm{~kb}$ for loblolly pine and $1 \mathrm{SSR} /$ $42.9 \mathrm{~kb}$ for spruce (Bérubé et al. 2007). Microsatellite genesis is due mainly to DNA slippage, unequal crossing over, gene conversion, and retro-transposition (Kalia et al. 2011). The frequencies of slippage and point mutations may affect distribution of SSRs in a genome. Also, the SSRs in genes contain higher mutation rates than nonrepetitive regions ( $\mathrm{Li}$ et al. 2004). The density and sequence numbers of EST-SSRs in Betula may be induced by mutation mechanisms and genome re-organization in evolutionary history.

In this study, the most abundant motif was found to be di-nucleotides $(124,65.9 \%)$. This is in agreement with the results of earlier studies of Arabidopsis (Cardle et al. 2000), C. sinensis (Shanker et al. 2007), and loblolly pine and spruce (Bérubé et al. 2007). These similar results might be related to SSRs in non-coding regions. Three-nucleotide repeats are an abundant motif in Betula and similar results were found in earlier studies, e.g. Lolium perenne (Asp et al. 2007), castor bean (Qiu et al. 2010), medicinal plant Ocimum basilicum (Gupta et al. 2010a, b), and Q. robur (Filiz et al. 2012). Toth et al. (2000) and Morgante et al. (2002) reported a higher frequency of GA/CT repeat than AT repeat in Arabidopsis and cereals and our findings (GA was the most frequent motif in Betula) were similar to these study results. In three-nucleotides, AAG in Arabidopsis, CCG in cereals (Varshney et al. 2002), AAG/ CTT in cotton (Lü et al. 2010), AAG/CTT in Q. robur (Filiz et al. 2012), AAG/CTT in castor bean (Qiu et al. 2010) were the most frequent motifs and the data supports our findings revealing AAG repeat $(3.7 \%)$ was the most abundant motif in Betula. The abundance of tri-nucleotide SSRs in coding regions may be related to the selective risk of non-trimeric SSR variants in coding regions, perhaps resulting from frame-shift mutations (Metzgar et al. 2000). 
Table 2 Details of SSR containing ESTs with significant matches in B. pendula, including accession numbers, repeat motif, primer sequences, product size, and annealing temperature

\begin{tabular}{|c|c|c|c|c|c|}
\hline No. & $\begin{array}{l}\text { Accession } \\
\text { number }\end{array}$ & Motif & Forward/reverse primer & $\begin{array}{l}\text { Product } \\
\text { size (bp) }\end{array}$ & $\begin{array}{l}\text { Annealing } \\
\text { temperature }\left({ }^{\circ} \mathrm{C}\right)\end{array}$ \\
\hline 1 & CD278929 & $(\mathrm{AG}) 8$ & GTGCGACGAGACACAGAGAG/TAAGCCAGTTGCGATGTCAG & 210 & 59.76 \\
\hline 2 & CD278925 & (CT) 12 & CATTCCTCGAATGGATTGCT/AGAAAAGGCGCAACACAACT & 195 & 59.92 \\
\hline 3 & CD278888 & $(\mathrm{CT}) 10$ & GCAGATTGAACACGCTTTGA/TTTTTCGAACCAAAACTCGAA & 209 & 60.00 \\
\hline 4 & CD278876 & $(\mathrm{CT}) 7$ & TTCAATCTTTAGGCGCGTTT/TAAAGGGCATTCCACAGGTC & 208 & 59.85 \\
\hline 5 & CD278819 & $(\mathrm{TCA}) 5$ & CGTCATCTCCGTGGACAATA/CAACCAGCCATACACACCAT & 202 & 59.29 \\
\hline 6 & CD278757 & $(\mathrm{AAG}) 6$ & CGACTCTGTGGAGTCATGGA/CGCCTTTATCTCCAGCTTTG & 211 & 59.82 \\
\hline 7 & $\mathrm{CD} 278750$ & (TCA) 5 & GGACTTCTTCGGAGACATGG/AATGTCGGAGTCGTCGAAGT & 209 & 59.73 \\
\hline 8 & CD278749 & (ATCTG) 4 & GGCGATATCCTTGAATCGAA/GTCTCGCGGTCGTTGATAAT & 194 & 60.10 \\
\hline 9 & CD278733 & $(\mathrm{AT}) 9$ & AAAACCGTGTCTCTCGCAGT/CCGGAAAATTTGGTCCACTA & 205 & 59.79 \\
\hline 10 & CD278714 & $(\mathrm{AG}) 10$ & TCTACGCAGACGCAGAGAGA/AGGAGGTTCCTTTCCTCCAC & 199 & 59.53 \\
\hline 11 & CD278710 & $(\mathrm{TCA}) 5$ & CGTCATCTCCGTGGACAATA/AACCAGCCAAACACACCATT & 201 & 59.52 \\
\hline 12 & CD278665 & $(\mathrm{GCGA}) 4$ & GAGCGAGCGAGAGACAGACT/CCCCTTTCTTCAGATCAACG & 206 & 59.66 \\
\hline 13 & CD278629 & $(\mathrm{CT}) 7$ & GCTCAAGTGGGTCCGTTTTA/ACCGCAGAAGCATACTCCAC & 204 & 60.29 \\
\hline 14 & $\mathrm{CD} 278585$ & (TC) 8 & TCAATGAGAATGGCGACAAA/GAATGCCGCAAGAGTTCAAT & 191 & 60.22 \\
\hline 15 & CD278562 & (GAT5) & TTCAGTTGATGCTGCTCCTG/TCTCATCATCCCAAGGCTTC & 203 & 60.16 \\
\hline 16 & CD278540 & $(\mathrm{CCGC}) 4$ & TGTGGAGGAGCATGTAGTGC/GGGCGGACATATGTTTCAAG & 195 & 59.86 \\
\hline 17 & CD278539 & $(\mathrm{CT}) 9$ & TGGGTCCAGACTTTCGAGTT/CATTGTCATCAAACCCAGCA & 192 & 59.70 \\
\hline 18 & CD278525 & $(\mathrm{TA}) 7$ & GTAGGCCGCCCAAAGACTAT/CGTTTCGTCCCAAATCTTGT & 170 & 59.97 \\
\hline 19 & $\mathrm{CD} 278521$ & $(\mathrm{GAT}) 5$ & AGACTCCTTTCCGAATGCAA/CGTCCTCTGTCTCATCACCA & 200 & 59.82 \\
\hline 20 & CD278493 & $(\mathrm{GAGC}) 5$ & GAGCGAGCGAGAGACAGACT/CCCCTTTCTTCAGATCAACG & 206 & 59.66 \\
\hline 21 & $\mathrm{CD} 278458$ & $(\mathrm{AT}) 8$ & CTCGACATGGGCTACACAGA/CAAGGCCTTCTCTGCTTCAA & 194 & 59.86 \\
\hline 22 & CD278435 & $(\mathrm{AAG}) 5$ & GAAGATGTCGTGGCAAACCT/ACCAAGGTACAGGCCAGTTG & 208 & 60.03 \\
\hline 23 & $\mathrm{CD} 278413$ & $(\mathrm{TC}) 7$ & GGCTCTTCCTGCTGATTCTG/CACTCCССTTCTTCTCAACG & 200 & 59.84 \\
\hline 24 & CD278341 & $(\mathrm{GAGC}) 5$ & GAGCGAGCGAGAGACAGACT/CCCCTTTCTTCAGATCAACG & 206 & 59.66 \\
\hline 25 & CD278329 & $(\mathrm{AG}) 7$ & TGGAGGGGTCATCTATCTCG/CCCСTCTCAACACCCATATC & 206 & 59.21 \\
\hline 26 & CD278320 & $(\mathrm{CT}) 7$ & CAAACCAGAGCCTCTCATCC/GCGTCCCTATAGGCATCATT & 202 & 59.03 \\
\hline 27 & CD278298 & (TC) 13 & TGATAGGGAGCGGATACCAG/ACGAGGATCCCTCAAGGTTT & 199 & 59.93 \\
\hline 28 & CD278287 & (ATG)5 & AAGATCGCCTTCGATGACAC/CCAATCGCCTACTTGACCAC & 199 & 60.52 \\
\hline 29 & CD278258 & (AG) 10 & AGAGCCGCTTCACAGAGAGA/GGTTTCCTCGTCGGTTATGA & 199 & 59.93 \\
\hline 30 & CD278220 & $(\mathrm{AG}) 12$ & GAAAGGGGCTTCATCAGTTG/TTTGAATTGAGCAGCCATCA & 199 & 59.67 \\
\hline 31 & $\mathrm{CD} 278175$ & $(\mathrm{AAG}) 5$ & GAAGATGTCGTGGCAAACCT/ACCAAGGTACAGGCCAGTTG & 208 & 60.12 \\
\hline 32 & CD278136 & $(\mathrm{AG}) 7$ & TGGAGGGGTCATCTATCTCG/TGGAGGGGTCATCTATCTCG & 206 & 59.21 \\
\hline 33 & CD278102 & (TGG)5 & ATGAAGGTGATCGCTGCATA/CCAGAAGGCACAGATGCTAA & 203 & 59.26 \\
\hline 34 & CD278084 & (TC) 11 & GCGACAGGAAATTCAACCAC/ACGTTCTGCTCCTTCAATCG & 211 & 60.40 \\
\hline 35 & CD278049 & (TGG)6 & TGCTCTCGTTTCCAACCTCT/CCCTTGACTTCACACAAGAGC & 195 & 59.90 \\
\hline 36 & CD277996 & $(\mathrm{CT}) 10$ & AAACATGTCGGCTTTCAAGG/AAACATGTCGGCTTTCAAGG & 200 & 60.66 \\
\hline 37 & CD277982 & $(\mathrm{AG}) 8$ & AGAGCAAGCCGAGAGATACG/CTCCTCGCATTCTGTTCGTT & 201 & 59.74 \\
\hline 38 & CD277916 & $(\mathrm{GAGC}) 5$ & GAGCGAGCGAGAGACAGACT/CCCCTTTCTTCAGATCAACG & 206 & 59.66 \\
\hline 39 & CD277897 & $(\mathrm{AG}) 8$ & AAGTGGCATTGGTCACAGGT/TGCACGGCATACATCATCTT & 197 & 60.43 \\
\hline 40 & CD277853 & $(\mathrm{GAT}) 5$ & CCTCGACGAGTTCCTCTCTG/TGAAGCACCTTTGCCATACA & 205 & 60.26 \\
\hline 41 & $\mathrm{CD} 277850$ & $(\mathrm{CT}) 14$ & TGATAGGGAGCGGATACCAG/ACGAGGATCCCTCAAGGTTT & 199 & 59.93 \\
\hline 42 & CD277824 & (TC) 8 & TTTTGGGCTCCATCTCATTC/TTGAGTCCCGTCCATTCTTT & 202 & 59.53 \\
\hline 43 & CD277797 & $(\mathrm{AG}) 8$ & GAAAGGGAAGCGAAGAGGAC/TCCTTATTGGGTGCATAGGG & 190 & 59.78 \\
\hline 44 & CD277764 & $(\mathrm{AAG}) 5$ & GAAGATGTCGTGGCAAACCT/ACCAAGGTACAGGCCAGTTG & 208 & 60.03 \\
\hline 45 & $\mathrm{CD} 277747$ & $(\mathrm{GA}) 8$ & GACTACCTCCGCTTCGTCAC/GAGCTACCTCGTCGTCGTTG & 202 & 59.87 \\
\hline 46 & CD277714 & $(\mathrm{GAGC}) 5$ & GAGCGAGCGAGAGACAGACT/CCGATTTTCTTAGGAGCGATG & 214 & 61.05 \\
\hline
\end{tabular}


Table 2 continued

\begin{tabular}{|c|c|c|c|c|c|}
\hline No. & $\begin{array}{l}\text { Accession } \\
\text { number }\end{array}$ & Motif & Forward/reverse primer & $\begin{array}{l}\text { Product } \\
\text { size (bp) }\end{array}$ & $\begin{array}{l}\text { Annealing } \\
\text { temperature }\left({ }^{\circ} \mathrm{C}\right)\end{array}$ \\
\hline 47 & $\mathrm{CD} 277683$ & $(\mathrm{TCA}) 5$ & CGTCATCTCCGTGGACAATA/AACCAGCCAAACACACCATT & 200 & 59.52 \\
\hline 48 & CD277622 & $(\mathrm{GAA}) 5$ & GAGCAGTGCCGGTTTATCTC/TGAATGTGAACCCAGGACAA & 189 & 59.94 \\
\hline 49 & CD277609 & $(\mathrm{TC}) 7$ & GGCTCTTCCTGCTGATTCTG/CACTCCССТTCTTCTCAACG & 200 & 59.84 \\
\hline 50 & CD277589 & (TC)13 & GCAAGCGTCTTTCAAGCATT/CCTTGATACCAGGGAGAACG & 201 & 59.54 \\
\hline 51 & $\mathrm{CD} 277535$ & $(\mathrm{CT}) 8$ & TTGGTGGTTGTTCTTGTCCA/CTCACAGACCCACTTGCAGA & 204 & 59.98 \\
\hline 52 & $\mathrm{CD} 277532$ & $(\mathrm{AAG}) 5$ & GAAGATGTCGTGGCAAACCT/ACCAAGGTACAGGCCAGTTG & 208 & 60.03 \\
\hline 53 & $\mathrm{CD} 277530$ & $(\mathrm{GAGC}) 4$ & GAGCGAGCGAGAGACAGACT/CCCCTTTCTTCAGATCAACG & 206 & 59.66 \\
\hline 54 & $\mathrm{CD} 277502$ & $(\mathrm{TC}) 7$ & ACGCTTTCGTGTTTCTTGCT/TTCTTCTTCCACGCTGATCC & 199 & 60.34 \\
\hline 55 & $\mathrm{CD} 277473$ & $(\mathrm{CT}) 9$ & CCAACAGGCTTTCATTTGCT/ACAAGAGCTCGGTTCTGGTC & 200 & 59.45 \\
\hline 56 & $\mathrm{CD} 277411$ & $(\mathrm{CTT}) 5$ & GAGATGGCGGAGACTGAGAC/TGGATGAAAAGCACAGGTTG & 200 & 59.69 \\
\hline 57 & $\mathrm{CD} 277408$ & $(\mathrm{AT}) 9$ & TGTAGCAGAGATGGCCTTGA/GGAATCCATGGCAAACCTTA & 199 & 59.76 \\
\hline 58 & $\mathrm{CD} 277395$ & $(\mathrm{TC}) 8$ & TTTTGGGCTCCATCTCATTC/TTGAGTCCCGTCCATTCTTT & 202 & 59.53 \\
\hline 59 & $\mathrm{CD} 277391$ & (TGG)5 & TGCTCTCGTTTCCAACCTCT/CCCTTGACTTCACACAAGAGC & 195 & 59.90 \\
\hline 60 & $\mathrm{CD} 277387$ & $(\mathrm{CT}) 8$ & TGGGTGCTCTGGACTTTCTC/TACTGTTACCCGGCTCTGCT & 194 & 59.90 \\
\hline 61 & CD277383 & $(\mathrm{AG}) 7$ & AGCTTCGTTCCAAAACCTCA/CCCATTTGGAGATGGAGAAA & 208 & 59.86 \\
\hline 62 & $\mathrm{CD} 277382$ & (TC) 11 & CGCAGAGTCTTCGACATGAG/TCACCACCATGCCAATATCA & 199 & 60.76 \\
\hline 63 & $\mathrm{CD} 277308$ & $(\mathrm{GGT}) 5$ & GACATGCAATCCCTTGGAGT/ACAACTTGGGGTGGAAACAC & 202 & 59.72 \\
\hline 64 & $\mathrm{CD} 277302$ & $(\mathrm{CT}) 11$ & AGAACTGTTGGGAGGTGCAG/CGTGGCTGAGTGAGGTTGTA & 204 & 59.90 \\
\hline 65 & $\mathrm{CD} 277285$ & (TC) 11 & CATGGTGGTGATCAGAGGAA/GGGCTAAAAATGGTCCACCT & 197 & 60.19 \\
\hline 66 & $\mathrm{CD} 277250$ & $(\mathrm{CT}) 12$ & CGAATTGAAGGAGCAGAAGG/TGCTCACAGCAAAGCAGAGT & 189 & 59.93 \\
\hline 67 & CD277249 & $(\mathrm{GATCC}) 4$ & CCTCCACCCGTTCAAGTGTA/TCACTTTGTGCACTGCCATT & 210 & 60.31 \\
\hline 68 & $\mathrm{CD} 277243$ & $(\mathrm{TC}) 9$ & CATTCCAGTCCATTCCGTTC/CCAATTTGTCAGCCGTATCA & 203 & 59.54 \\
\hline 69 & $\mathrm{CD} 277238$ & $(\mathrm{TCA}) 5$ & CGTCATCTCCGTGGACAATA/AACCAGCCAAACACACCATT & 201 & 59.52 \\
\hline 70 & CD277237 & $(\mathrm{ATG}) 6$ & AAGATCGCCTTCGATGACAC/CCAATCGCCTACTTGACCAC & 199 & 60.52 \\
\hline 71 & CD277232 & $(\mathrm{CT}) 14$ & GACTACCAACTCCGGTGCTC/TCATGGGTGACCTCAAAGAA & 189 & 59.06 \\
\hline 72 & CD277148 & $(\mathrm{AG}) 10$ & GGAGTACAGGCAGAGGGTTG/CAGTGACTGATCCCCAGCTT & 199 & 59.72 \\
\hline 73 & CD277140 & $(\mathrm{CT}) 8$ & TGGGTGCTCTGGACTTTCTC/TACTGTTACCCGGCTCTGCT & 194 & 59.90 \\
\hline 74 & CD277125 & $(\mathrm{CT}) 11$ & AGAACTGTTGGGAGGTGCAG/CGTGGCTGAGTGAGGTTGTA & 204 & 59.90 \\
\hline 75 & CD277116 & $(\mathrm{CT}) 7$ & AATTCGGTGGGGGACTAGAG/CATTCACAAGGACCAGAACG & 195 & 59.13 \\
\hline 76 & CD277113 & $(\mathrm{TC}) 9$ & GCCGCTTTGAGACTCTGATT/GGGACATAGGTTGCATGCTT & 202 & 59.96 \\
\hline 77 & CD277098 & $(\mathrm{GAA}) 5$ & GGCGTTTAATCTGGGTGAGA/TTCCTGATGTCGAATGCTCA & 213 & 60.35 \\
\hline 78 & CD277085 & $(\mathrm{GA}) 9$ & CTGGCAAAAGTGTGCAGAAA/CTGAGCATCAAGTGCCAAAG & 191 & 59.59 \\
\hline 79 & CD277013 & $(\mathrm{AG}) 11$ & TGGTTGAAGCGATGAAGACA/CAACCCTCTGCCTGTACTCC & 201 & 59.72 \\
\hline 80 & CD276959 & (TC) 10 & AATTCGGTGGGGGACTAGAG/CATTCACAAGGACCAGAACG & 195 & 59.13 \\
\hline 81 & CD276953 & $(\mathrm{CT}) 8$ & TCСАТTTCСТCAАСССТСТC/TGAGAGCССТССТTTСТTCA & 199 & 59.06 \\
\hline 82 & CD276933 & $(\mathrm{TC}) 7$ & TGCTCCGGTTTACAACAATG/CGAAGCGATAGTGACGACAG & 192 & 59.62 \\
\hline 83 & CD276907 & $(\mathrm{CT}) 8$ & TTGGTGGTTGTTCTTGTCCA/CTCACAGACCCACTTGCAGA & 204 & 59.98 \\
\hline 84 & CD276864 & $(\mathrm{GAT}) 5$ & TTCAGTTGATGCTGCTCCTG/TCTCATCATCCCAAGGCTTC & 203 & 60.16 \\
\hline 85 & CD276856 & $(\mathrm{CT}) 9$ & AGCGCTCCCTCTCTCTCTCT/TTAGCTCCCACCACGTTAGC & 205 & 60.27 \\
\hline 86 & CD276840 & $($ GATCC) 4 & CAGCTCCTTTGGACTCTTCG/TAAGGCACACGATCTGCTTG & 183 & 60.01 \\
\hline 87 & CD276777 & $(\mathrm{TCT}) 6$ & CGGCTTTCTCTCССТCTCTT/ATGACCACAGCGAACACTTG & 204 & 59.75 \\
\hline 88 & CD276759 & (TC) 10 & CGCAGAGTCTTCGACATGAG/TCACCACCATGCCAATATCA & 199 & 59.73 \\
\hline 89 & CD276730 & $(\mathrm{CT}) 7$ & TTGGTGGTTGTTCTTGTCCA/CTCACAGACCCACTTGCAGA & 204 & 59.98 \\
\hline 90 & CD276682 & (ATCTG)4 & CCCTATGGCGATATCCTTGA/GTCTCGCGGTCGTTGATAAT & 200 & 59.88 \\
\hline 91 & CD276680 & $(\mathrm{GAT}) 5$ & CCTCGACGAGTTCCTCTCTG/TGAAGCACCTTTGCCATACA & 205 & 60.26 \\
\hline 92 & CD276664 & $(\mathrm{TCT}) 6$ & CGCCAAATCTTTACCCAGAA/CATCTCGATCCTCTCCTTCG & 208 & 59.90 \\
\hline 93 & CD276628 & $(\mathrm{CT}) 8$ & GGTGACTTGGTGGGTGTTCT/CCCACTTGCAGACAGCAATA & 202 & 59.86 \\
\hline
\end{tabular}


Table 2 continued

\begin{tabular}{|c|c|c|c|c|c|}
\hline No. & $\begin{array}{l}\text { Accession } \\
\text { number }\end{array}$ & Motif & Forward/reverse primer & $\begin{array}{l}\text { Product } \\
\text { size (bp) }\end{array}$ & $\begin{array}{l}\text { Annealing } \\
\text { temperature }\left({ }^{\circ} \mathrm{C}\right)\end{array}$ \\
\hline 94 & CD276623 & $(\mathrm{TC}) 7$ & AGTGCTATGGCGAAGGACAT/TCGGACTGGCTCTTGTATCC & 206 & 59.72 \\
\hline 95 & CD276610 & (TCT)6 & TCGCTGTGGTCATGATAGGA/ATCTTTGCCCTGCTCTTCAA & 206 & 59.96 \\
\hline 96 & CD276602 & $($ GGAA) 4 & GGTTTGTGCCAACGAAACTT/GGCAAGACCTTCCTTCCTTC & 197 & 60.19 \\
\hline 97 & CD276578 & $(\mathrm{AT}) 8$ & CTCGACATGGGCTACACAGA/GTCAAGGCCATCTCTGCTTC & 196 & 59.96 \\
\hline 98 & CD276494 & (TCT)6 & CGGCTTTCTCTCССТCTCTT/ATGACCACAGCGAACACTTG & 204 & 59.75 \\
\hline 99 & Contig 8 & $(\mathrm{AAG}) 5$ & GAAGATGTCGTGGCAAACCT/ACCAAGGTACAGGCCAGTTG & 208 & 60.03 \\
\hline 100 & Contig29 & $(\mathrm{GAGC}) 4$ & TGGAGCAGATGAAGCAACAC/TGGAGCAGATGAAGCAACAC & 203 & 59.97 \\
\hline 101 & Contig94 & $(\mathrm{GAT}) 5$ & CCTCGACGAGTTCCTCTCTG/TGAAGCACCTTTGCCATACA & 205 & 60.26 \\
\hline 102 & Contig102 & $(\mathrm{AAG}) 5$ & GAAGATGTCGTGGCAAACCT/ACCAAGGTACAGGCCAGTTG & 208 & 60.12 \\
\hline
\end{tabular}

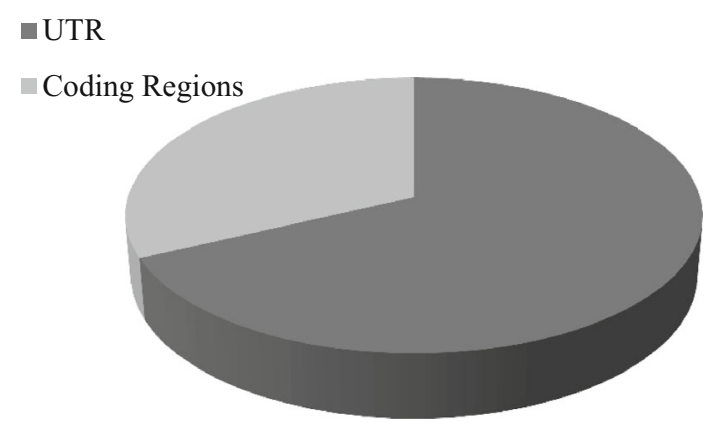

Fig. 3 Distribution of EST-SSRs in coding regions and UTRs

It was observed that serine $(19.6 \%)$ was found to be the most frequent amino acid encoded by trinucleotide SSRs. The most common amino acid was found to be serine (Ser) in Quercus and Arabidopsis (Lawson and Zhang 2006; Filiz et al. 2012) and these data are in agreement with our results. However, lysine (Lys) in Arabidopsis (Morgante et al. 2002) and arginine (Arg) in sugarcane (Cordeiro et al. 2001) were found to be the most encoded amino acids and these results contradict our findings. SSRs in promoter and intronic regions may affect gene activity and gene transcription. Also, SSR repeat numbers may regulate gene expression and expression level ( $\mathrm{Li}$ et al. 2002). Lately, new evidence shows that large numbers of SSRs are located in coding regions of genomes (Morgante et al. 2002), suggesting that the difference in the distribution of amino acids could be a result of different gene expression profiles that affect SSRs in coding regions. In Arabidopsis, high densities of SSRs were found to be in UTRs (Lawson and Zhang 2006). Similar results were reported for the medicinal plant Ocimum basilicum (Gupta et al. 2010a, b) and for $C$. sinensis (Shanker et al. 2007). These data corroborated our results that nearly $68 \%$ of SSRs were located in UTR regions in Betula. It can be proposed that SSRs in UTR regions play important roles in gene regulation. 102 of 178 EST-SSRs (57.3\%) were annotated and categorized into different classes, including ribosomal protein, transport protein, membrane protein, carrier protein, binding protein, transferase protein, and others. Also, 102 primers were designed for these putative proteins. In conclusion, ESTs of $B$. pendula were mined for EST-SSRs and the current study demonstrated that understanding of SSR distribution could support future studies with in silico comparative analysis of Betulaceae species, especially Betula taxa. Also, EST-SSR resources developed from this work can be utilized in studies of genetic diversity, linkage mapping, and comparative analyses in Betulaceae species.

\section{References}

Acuña CV, Fernandez P, Villalba PV, García MN, Hopp HE, Poltri SNM (2011) Discovery, validation, and in silico functional characterization of EST-SSR markers in Eucalyptus globules. Tree Genet Genomes. doi:10.1007/s11295-011-0440-0

Asp T, Frei UK, Didion T, Nielsen KK, Lübberstedt T (2007) Frequency, type, and distribution of EST-SSRs from three genotypes of Lolium perenne, and their conservation across orthologous sequences of Festuca arundinacea, Brachypodium distachyon, and Oryza sativa. BMC Plant Biol 7:36. doi:10. 1186/1471-2229-7-36

Bérubé Y, Zhuang J, Rungis D, Ralph S, Bohlmann J, Ritland K (2007) Characterization of EST-SSRs in loblolly pine and spruce. Tree Genet Genomes 3:251-259

Cardle L, Ramsay L, Milborne D, Macaulay M, Marshall D, Waugh R (2000) Computational and experimental characterization of physically clustered simple sequence repeats in plants. Genetics 156:847-854

Cordeiro GM, Casu R, McIntyre CL, Manners JM, Henry RJ (2001) Microsatellite markers from sugarcane (Saccharum spp.) ESTs cross transferable to erianthus and sorghum. Plant Sci 160: $1115-1123$

De Jong PC (1993) An introduction to Betula: its morphology, evolution, classification and distribution with a survey of recent work. The IDS Betula Symposium, International Dendrology Society, Susses 
Ellegren H (2000) Microsatellite mutations in the germline: implications for evolutionary inference. Trends Genet 16:551-558

Filiz E, Koc I, Sakinoglu FC (2012) In silico EST-SSRs analysis in unigene of Quercus robur L. Res Plant Biol 2:1-9

Furlow JJ (1990) The genera of Betulaceae in the southeastern United States. J Arnold Arbor 71:1-67

Gupta PK, Balyan HS, Sharma PC, Ramesh B (1996) Microsatellites in plants: a new class of molecular markers. Curr Sci 70:45-54

Gupta PK, Rustgi S, Sharma S, Singh R, Kumar N, Balyan HS (2003) EST-SSRs for transferability, polymorphism and genetic diversity in bread wheat. Mol Genet Genom 270:315-323

Gupta S, Shukla R, Roy S, Sen N, Sharma A (2010a) In silico SSR and FDM analysis through EST sequences in Ocimum basilicum. POJ 3:121-128

Gupta S, Tripathi KP, Roy S, Sharma A (2010b) Analysis of unigene derived microsatellite markers in family Solanaceae. Bioinformation 5:113-121

Huang X, Madan A (1999) CAP3: a DNA sequence assembly program. Genome Res 9:868-877

Kalia RK, Rai MK, Kalia S, Singh R, Dhawan AK (2011) Microsatellite markers: an overview of the recent progress in plants. Euphytica 177:309-334

Kota R, Varshney RK, Thiel T, Dehmer KJ, Graner A (2001) Generation and comparison of EST-derived SSRs and SNPs in barley (Hordeum vulgare L.). Hereditas 135:145-151

Lawson MJ, Zhang L (2006) Distinct patterns of SSR distribution in the Arabidopsis thaliana and rice genomes. Genome Biol 7:R14

Li YC, Korol AB, Fahima T, Beiles A, Nevo E (2002) Microsatellites: genomic distribution, putative functions and mutational mechanisms: a review. Mol Ecol 11:2453-2465

Li YC, Korol AB, Fahima T, Nevo E (2004) Microsatellites within genes: structure, function, and evolution. Mol Biol Evol 21: 991-1007

Lü YD, Cai CP, Wang L, Lin SY, Zhao L, Tian LL, Lü JH, Zhang TZ, Guo WZ (2010) Mining, characterization, and exploitation of EST-derived microsatellites in Gossypium barbadense. Chin Sci Bull 55:1889-1893

Martín C, Parra T, Clemente-Muñoz M, Hernandez-Bermejo E (2008) Genetic diversity and structure of the endangered Betula pendula subsp. fontqueri populations in the south of Spain. Silva Fenn 42:487-498
Metzgar D, Bytof J, Wills C (2000) Selection against frameshift mutations limits microsatellite expansion in coding DNA. Genome Res 10:72-80

Morgante M, Hanafey M, Powell W (2002) Microsatellite are preferentially associated with nonrepetitive DNA in plant genomes. Nat Genet 30:194-200

Palliyarakkal MK, Ramaswamy M, Vadivel A (2011) Microsatellites in palm (Arecaceae) sequences. Bioinformation 7:347-351

Powell W, Machray GC, Provan J (1996) Polymorphism revealed by simple sequence repeats. Trends Plant Sci 1:215-222

Qiu L, Yang C, Tian B, Yang JB, Liu A (2010) Exploiting EST databases for the development and characterization of EST-SSR markers in castor bean (Ricinus communis L.). BMC Plant Biol 10:278

Rungis D, Bérubé Y, Zhuang J, Ralph S, Ritland CE, Ellis BE, Douglas C, Bohlmann J, Ritland K (2004) Robust simple sequence repeat (SSR) markers for spruce (Picea spp.) from expressed sequence tags (ESTs). Theor Appl Genet 109: 1283-1294

Schenk MF, Thienpont CN, Koopman WJM, Gilissen LJWJ, Smulders MJM (2008) Phylogenetic relationships in Betula (Betulaceae) based on AFLP markers. Tree Genet Genomes 4:911-924

Scott KD, Eggler P, Seaton G, Rossetto M, Ablett EM, Lee LS, Henry RJ (2000) Analysis of SSRs derived from grape ESTs. Theor Appl Genet 100:723-726

Shanker A, Bhargava A, Bajpai R, Singh S, Srivastava S, Sharma V (2007) Bioinformatically mined simple sequence repeats in UniGene of Citrus sinensis. Sci Hortic 113:353-361

Tautz D (1993) Notes on the definition and nomenclature of tandemly repetitive DNA sequences. In: Pena SDJ, Chakraborty R, Epplen JT, Jeffreys AJ (eds) DNA fingerprinting: state of science. Birkhäuser Verlag, Basel, pp 21-28

Tautz D, Renz M (1984) Simple sequence repeats are ubiquitous repetitive components of eukaryotic genomes. Nucleic Acids Res 12:4127-4138

Toth G, Gáspári Z Z, Jurka J (2000) Microsatellites in different eukaryotic genomes: survey and analysis. Genome Res 10: 967-981

Varshney RK, Thiel T, Stein N, Langridge P, Graner A (2002) In silico analysis on frequency and distribution of microsatellites in ESTs of some cereal species. Cell Mol Biol Lett 7:537-546 